\title{
Systematic Search of Exactly Solvable Non-Central Potentials
}

\author{
B. Gönül and M. Koçak \\ Department of Engineering Physics, Faculty of Engineering, \\ University of Gaziantep, 27310 Gaziantep -Türkiye
}

\begin{abstract}
Recently developed supersymmetric perturbation theory has been successfully employed to make a complete mathematical analysis of the reason behind exact solvability of some non-central potentials. This investigation clarifies once more the effectiveness of the present formalism.
\end{abstract}

Keywords: Non-central potentials, Supersymmetry, Exact solvability PACS No: 03.65.Fd

\section{Introduction}

Using the basic ingredient of supersymmetry, a simple alternative approach has been recently put forward with its application to perturbed Coulomb potentials [1] in arbitrary dimensions, and subsequently a general procedure within this novel framework has been given for the exact treatment of quantum states having nonzero angular momenta [2], together with other work [3] in which it has been clearly shown that approaches based on logarithmic and supersymmetric perturbation theories are involved within the more general framework of the formalism.

Gaining confidence from these works we aim with this Letter to illustrate the idea of this basic but powerful technique can also be readily used to search exact solvability of non-central potentials, which clarifies the systematic behind such algebraic treatments.

Analytically solvable potentials are important for a number of reasons such as providing model problems to analyze, to start perturbation theory expansions from, or to provide complete sets of basis functions for solving real problems. In this respect, using the ideas of supersymmetry and shape invariance [4], many authors [5]-[8] obtained the solutions of a wide class of non-central potentials in a closed form. Additionally, in a recent work [9] similar techniques have been 
used to determine the spectrum of a vibrational molecular system and using some well-known shape invariant potentials the authors have obtained energy levels of triatomic molecules for 12 classes of non-central but separable potentials. Nevertheless, to the best of our knowledge, the answer of the natural question that why some of the non-central potentials can be solved exactly has not been discussed in the literature, which is the task of the present work.

\section{The Model}

We first start with a brief introduction of the present formalism. Throughout the paper the unit system $\hbar=2 m=1$ is chosen. The goal in the supersymmetric quantum theory is to solve the Riccati equation,

$$
W^{2}(r)-W^{\prime}(r)=V(r)-E_{0},
$$

where $V(r)$ is the potential of interest and $E_{0}$ is the corresponding ground state energy. If we find $W(r)$, the so called superpotential, we have of course found the ground state wave function via,

$$
\Psi_{0}(r)=N \exp \left[-\int W(z) d z\right]
$$

where $N$ is the normalization constant. If $V(r)$ is a shape invariant potential,we can in fact obtain the entire spectrum of bound state energies and wave functions via the ladder operators [4]. Now, suppose that we are interested in a potential for which we do not know $W(r)$ exactly. More specifically, we assume that $V(r)$ differs by a small amount from a potential $V_{0}(r)$ plus angular momentum barrier if any, for which one solves the Riccati equation explicitly. For the consideration of spherically symmetric potentials, the corresponding Schrödinger equation for the radial wave function has the form

$$
\frac{\Psi_{n}^{\prime \prime}(r)}{\Psi_{n}(r)}=\left[V(r)-E_{n}\right], \quad V(r)=\left[V_{0}(r)+\frac{\ell(\ell+1)}{r^{2}}\right]+\Delta V(r)
$$

where $\Delta V$ is a perturbing potential. Let us write the wave function $\Psi_{n}$ as

$$
\Psi_{n}(r)=\chi_{n}(r) \phi_{n}(r)
$$

in which $\chi_{n}$ is the known normalized eigenfunction of the unperturbed Schrödinger equation whereas $\phi_{n}$ is a moderating function corresponding to the perturbing potential. Substituting (4)into (3) yields

$$
\left(\frac{\chi_{n}^{\prime \prime}}{\chi_{n}}+\frac{\phi_{n}^{\prime \prime}}{\phi_{n}}+2 \frac{\chi_{n}^{\prime}}{\chi_{n}} \frac{\phi_{n}^{\prime}}{\phi_{n}}\right)=V-E_{n}
$$


Instead of setting the functions $\chi_{n}$ and $\phi_{n}$, we will set their logarithmic derivatives using the spirit of Eqs.(1) and (2);

$$
W_{n}=-\frac{\chi_{n}^{\prime}}{\chi_{n}}, \quad \Delta W_{n}=-\frac{\phi_{n}^{\prime}}{\phi_{n}}
$$

which leads to

$$
\frac{\chi_{n}^{\prime \prime}}{\chi_{n}}=W_{n}^{2}-W_{n}^{\prime}=\left[V_{0}(r)+\frac{\ell(\ell+1)}{r^{2}}\right]-\varepsilon_{n},
$$

where $\varepsilon_{n}$ is the eigenvalue of the unperturbed and exactly solvable potential, and

$$
\left(\frac{\phi_{n}^{\prime \prime}}{\phi_{n}}+2 \frac{\chi_{n}^{\prime}}{\chi_{n}} \frac{\phi_{n}^{\prime}}{\phi_{n}}\right)=\Delta W_{n}^{2}-\Delta W_{n}^{\prime}+2 W_{n} \Delta W_{n}=\Delta V(r)-\Delta \varepsilon_{n}
$$

in which $\Delta \varepsilon_{n}$ is the eigenvalue for the perturbed potential, and $E_{n}=\varepsilon_{n}+\Delta \varepsilon_{n}$. Then Eq.(5), and subsequently Eq.(3), reduces to

$$
\left(W_{n}+\Delta W_{n}\right)^{2}-\left(W_{n}+\Delta W_{n}\right)^{\prime}=V-E_{n},
$$

which is similar to Eq. (1). In principle as one knows explicitly the solution of Eq. (7), namely the whole spectrum and corresponding eigenfunctions of the unperturbed interaction potential, the goal here is to solve only Eq. (8), which is the backbone of this formalism, leading to the solution of Eqs. (3) and (9).

Although this is not the case in this paper, if the whole potential has no analytical solution, which means $\Delta W$ and subsequently Eq. (8) cannot be exactly solvable, then one can expand the functions in terms of the perturbation parameter $\lambda$,

$$
\begin{gathered}
\Delta V(r ; \lambda)=\sum_{k=1}^{\infty} \lambda^{k} \Delta V_{k}(r), \quad \Delta W_{n}(r ; \lambda)=\sum_{k=1}^{\infty} \lambda^{k} \Delta W_{n k}(r), \\
\Delta \varepsilon_{n}(\lambda)=\sum_{k=1}^{\infty} \lambda^{k} \varepsilon_{n k}
\end{gathered}
$$

where $\lambda$ will eventually be set equal to one. Substitution of the above expansion into Eq. (8) by equating terms with the same power of $\lambda$ on both sides yields up to $O\left(\lambda^{3}\right)$

$$
\begin{gathered}
2 W_{n} \Delta W_{n 1}-\Delta W_{n 1}^{\prime}=\Delta V_{1}-\Delta \varepsilon_{n 1}, \\
\Delta W_{n 1}^{2}+2 W_{n} \Delta W_{n 2}-\Delta W_{n 2}^{\prime}=\Delta V_{2}-\Delta \varepsilon_{n 2} \\
2\left(W_{n} \Delta W_{n 3}+\Delta W_{n 1} \Delta W_{n 2}\right)-\Delta W_{n 3}^{\prime}=\Delta V_{3}-\Delta \varepsilon_{n 3},
\end{gathered}
$$

Eq. (8) and its expansion, Eqs. (11-13), give a flexibility for the easy calculations of the perturbative corrections to energy and wave functions for the state of interest through an appropriately chosen perturbed superpotential, unlike the other perturbation theories. It has been shown [3] that this feature of the present model leads to a simple framework in obtaining the corrections to all states without using complicated and tedious mathematical procedures. 


\section{Application}

For the consideration of exactly solvable non-central potentials,

$$
U(r, \theta, \varphi)=U_{1}(r)+\frac{U_{2}(\theta)}{r^{2}}+\frac{U_{3}(\varphi)}{r^{2} \sin ^{2}(\theta)}
$$

the time-independent Schrödinger equation reads

$$
\begin{gathered}
\frac{d^{2} R}{d r^{2}}+\frac{2}{r} \frac{d R}{d r}+\left[E-U_{1}-\frac{\ell(\ell+1)}{r^{2}}\right] R=0, \\
\frac{d^{2} P}{d \theta^{2}}+\cot \theta \frac{d P}{d \theta}+\left[\ell(\ell+1)-\frac{m^{2}}{\sin ^{2}(\theta)}-U_{2}\right] P=0 \\
\frac{d^{2} \phi}{d \varphi^{2}}+\left(m^{2}-U_{3}\right) \phi=0
\end{gathered}
$$

where the total wavefunction is $\Psi(r, \theta, \varphi)=R(r) P(\theta) \phi(\varphi)$ and $\ell=0,1,2, \ldots, n-$ 1 together with $m=0, \pm 1, \ldots, \pm \ell$ are respectively the orbital and azimuthal quantum numbers. Here, the crucial point is that each part of the physically total interaction potential, namely $U_{1}, U_{2}$ and $U_{3}$, should be analytically solvable. As Eqs. (15) and (17), having an exactly solvable potential, were well discussed

in the literature based in particular on the supersymmetric quantum theory [4], we apply the technique presented in the previous section to only Eq. (16) to discuss the systematic behind such equations. However, one should bear in mind that the same procedure also can be employed easily in Eqs. (15) and (17), if necessary.

To proceed we use a mapping function $\theta=f(z)$ which transforms Eq. (16) into

$$
\frac{d^{2} P}{d z^{2}}+\left(-\frac{f^{\prime \prime}}{f^{\prime}}+f^{\prime} \cot f\right) \frac{d P}{d z}+f^{\prime 2}\left[\ell(\ell+1)-\frac{m^{2}}{\sin ^{2} f}-U_{2}(f)\right] P=0 .
$$

The aim here is to have a Schrödinger-like equation, therefore the second term above is removed with the choice of $\theta \equiv f=2 \tan ^{-1}\left(e^{z}\right)$ which yields $\sin \theta=$ $\sec h z, \cos \theta=-\tanh z$, leading to

$$
-\frac{d^{2} P}{d z^{2}}+\left[U_{2}(z)-\ell(\ell+1) \sec h^{2} z\right] P=-m^{2} P
$$

Now, the question is which forms of $U_{2}$ reproduce analytical solutions. To answer this question one needs to use the discussion given by Eqs. (3) through (9). As the whole interaction potential is,

$$
V(z)=V_{0}(z)+\Delta V(z)=-\ell(\ell+1) \sec h^{2} z+U_{2}(z)
$$


in case the angular part of the potential $U_{2}=0$ in (19), the remain piece leads to the well-known shape invariant exactly solvable potential [4]. It can be readily solved by the supersymmetric quantum theory,

$$
W_{n=0}(z)=\ell \tanh z, \quad \varepsilon_{n}=-(\ell-n)^{2}, \quad n=0,1,2, \ldots
$$

where $W_{n=0}$ and $\varepsilon_{n}$ denote respectively the superpotential and energy eigenvalue for the unperturbed potential, $V_{0}=-\ell(\ell+1) \sec h^{2} z$. Note that the corresponding wavefunctions reproduce standard properties of the spherical harmonics [6].

At this stage, with the consideration of Eq. (8)

$$
\Delta W_{n=0}^{2}(z)-\Delta W_{n=0}^{\prime}(z)+2(\ell \tanh z) \Delta W_{n=0}(z)=\Delta V(z)-\Delta \varepsilon_{n=0},
$$

one arrives at

$$
\Delta W_{n=0}=\frac{b}{\ell}, \quad \Delta \varepsilon_{n=0}=-\frac{b^{2}}{\ell^{2}}, \quad \Delta V(z)=2 b \tanh z,
$$

where $b$ is a constant. It makes clear that the full interaction potential has now the form of the Rosen-Morse II potential. A brief study of all shape invariant exactly solvable potentials [4] in one dimensional space, together with [7] related to non-central potentials, clarify the physically meaningful choice of $b$ as to be $b=-\frac{\gamma}{2}$ with $\gamma$ be another constant relating to the system of interest. For the completeness, one should now take consider the whole superpotential,

$$
W_{n=0}^{\text {total }}(z)=W_{n=0}(z)+\Delta W_{n=0}(z)=\ell \tanh z-\frac{\gamma}{2 \ell},
$$

which will reproduce the whole spectrum. Proceeding within the framework of supersymmetric quantum mechanics, the energy spectrum for the total potential $V=-\ell(\ell+1) \sec h^{2} z-\gamma \tanh z$ is given in the form

$$
E_{n}=-(\ell-n)^{2}-\frac{\gamma^{2}}{4(\ell-n)^{2}}=\varepsilon_{n}+\Delta \varepsilon_{n}
$$

which is the proof of the present theory. To see the exactly solvable form of the angular potential $U_{2}$, we use inverse mapping $\sec h z=\sin \theta, \tanh z=-\cos \theta$ and bearing in mind Eqs. (14) and (18), then arrives at

$$
U_{2}(\theta)=\frac{\gamma \cos \theta}{r^{2} \sin ^{2}(\theta)} .
$$

From the mathematical point of view, $r^{2} \sin ^{2} \theta$ in the calculations of exactly solvable forms of $U_{2}$ comes naturally, see Eqs. (14) and (18). Hence one can generalize the above potential involving a constant, if necessary, related to the physical system considered. In this case, such potentials are given as

$$
U_{2}(\theta)=\frac{\beta+\gamma \cos \theta}{r^{2} \sin ^{2}(\theta)}
$$


in which $\beta$ appears, from Eq. (18), only on RHS of Eq. (19) as a piece of energy value. Considering Eqs. (19) and (25) for the presence of $\beta$, we obtain

$$
\ell=n+\left[\frac{\left(m^{2}+\beta\right)+\sqrt{\left(m^{2}+\beta^{2}\right)-\gamma^{2}}}{2}\right]^{\frac{1}{2}} .
$$

This $\ell$ - value is used in the energy expression given for the central potential $U_{1}(r)$ to introduce the complete spectrum of analytically solvable non-central potentials. The present result agrees with Eq. (10) of Ref.[7], where the generalized Coulomb potential was discussed.

Clearly, Eq. (22) is the most significant equation of this Letter, which defines exactly the possible forms of $U_{2}(\theta)$ yielding analytical solutions. The main point here of course is to be able to find an analytical expression for $\Delta W$ and subsequently $\Delta V$ for the definition of the total $\theta$-dependent perturbing potential, which requires exactly the satisfaction of Eq. (22) leading to a closed form for the complete spectrum.

One should however note that although we have focussed here on the eigenvalues, calculation of the corresponding eigenfunctions within the same model is quite straightforward, see Eq. (2). In addition, we believe that this generalization would considerably extend the list of exactly solvable non-central potentials for which the solution can be obtained algebraically in a simple and elegant manner as discussed here.

A similar study is conducted here for the shape invariant Pösch-Teller II type potential [4], within the frame of Eqs. (19) through (22). This choice requires

$$
\Delta W_{n=0}(z)=-\alpha \operatorname{coth} z,
$$

which, from (22), reproduces

$$
\Delta V(z)=U_{2}(z)=\alpha(\alpha-1) \csc ^{2} z \quad, \quad \Delta \varepsilon_{n=0}=-\alpha(\alpha-2 \ell) .
$$

Thus, the full angle dependent potential in (19) turns into $V=-\ell(\ell+1) \sec h^{2} z+$ $\alpha(\alpha-1) \csc h^{2} z$ having a complete spectrum in the form of $E_{n}=-(\ell-\alpha-2 n)^{2}=$ $\varepsilon_{n}+\Delta \varepsilon_{n}$. Using the same algebraic procedure as before, one obtains the related exactly solvable non-central potential as

$$
U_{2}(\theta)=\frac{\delta}{r^{2} \sin ^{2}(\theta)}+\frac{c}{r^{2} \cos ^{2}(\theta)},
$$

in which $\delta$ and $c=\alpha(\alpha-1)$ are constants. As explained in the previous example, $\delta$ appears as a piece of energy eigenvalue on RHS of Eq. (19), therefore

$$
m^{2}+\delta=(\ell-\alpha-2 n)^{2}
$$


from which one defines the $\ell$ - value as

$$
\ell=2 n+\left(\frac{1}{2} \pm \sqrt{\frac{1}{4}+c}\right)+\left(m^{2}+\delta\right)^{\frac{1}{2}},
$$

that is the same result with compared to (26) of [7], and also agrees with the related references in [7]. If $U_{1}(r)$ is taken as the harmonic oscillator potential, then the whole non-central potential with $U_{3}(\varphi)=0$ corresponds to the generalized oscillatory potential. To find the full spectrum for such a potential, Eq. (33) is invoked to the energy spectrum of $U_{1}(r)[7]$.

\section{Concluding Remarks}

In this Letter, we have attempted to explore the effectiveness of the recently developed formalism through which we have made successfully the complete mathematical analysis of the reason behind exact solvability of some Schrödinger equations with a class of non-central but separable potentials, for which the complete spectrum and eigenfunctions can be written down algebraically using the well known results for the shape invariant potentials. Generalization of our technique to other non-central potentials is quite straightforward and use of the present model may also be useful for solving other quantum mechanical complicated systems analytically. With the above consideration the authors hope to stimulate further examples of applications of the model in important problems of physics,

which requires further technical work that will be discussed in a forthcoming paper.

\section{References}

[1] Özer O and Gönül B 2003 Mod.Phys. Lett. A18, 2581.

[2] Gönül B 2004 Chinese Phys. Lett. 21(appear in No.9); quant-ph/0407004.

[3] Gönül B 2004 submitted to Ann. Physik; quant-ph/0407003.

[4] Cooper F, Khare A and Sukhatme U P 1995 Phys. Rep. 251, 267.

[5] Khare A and Bhaduri R K 1994 Am. J. Phys. 62, 1008.

[6] Dutt R, Gangopadhyaya A and Sukhatme U P 1997 Am. J. Phys. 65, 400.

[7] Gönül B and Zorba İ 2000 Phys. Lett. A269, 83.

[8] Koçak M, Zorba İ and Gönül B 2002 Mod. Phys. Lett. A17, 2127.

[9] Hounkonnou M N, Sodoga K, Justum Y and Lauvergnat D 2004 J. Phys. B37, 1859. 